\title{
Status of Lipid Profile among the Hypertensive Patients in Bangladesh
}

\section{M REZA QURESHI FORHAD ${ }^{1}$, ASMA KABIR ${ }^{1}$, TUHIN BISWAS ${ }^{2}$, KAMRUN NAHAR CHOUDHURY $^{3}$, MD. ZAHIDUR RAHMAN ${ }^{4}$, DEENAAS HUSSAIN ${ }^{5}$, SHANTANU KUMAR GHOSH ${ }^{6}$}

\author{
${ }^{1}$ Tairunnessa Memorial Medical College and Hospital, ${ }^{2}$ International Research Centre for Diarrheal Diseases in Bangladesh, \\ ${ }^{3}$ National Centre for Control of Rheumatic fever and heart disease, ${ }^{4}$ The State Medical Faculty of Bangladesh, ${ }^{5}$ Shaheed Mansur \\ Ali Medical College, ${ }^{6}$ Bangabandhu Sheik Mujib Medical University \\ Address for Correspondence: Dr. C. M Reza Qureshi Forhad, Assistant Professor \& Head, Dept. of Biochemistry, Tairunnessa \\ Memorial Medical College, Targach, Board Bazar, Gazipur, Bangladesh, E-mail: forhad_reza@hotmail.com
}

\begin{abstract}
:
Back ground: Hypertension and dyslipidemia are associated with oxidative stress and are major causes of cardiovascular disease amounting to $30 \%$ of global death rate. It is widely accepted that cardiovascular disease is associated with hypertension and increased blood levels of low-density lipoprotein (LDL), total cholesterol (TC), and triglycerides. In contrast, a low level of high density lipoprotein (HDL) is a risk factor for mortality from cardiovascular disease. Hypertension is a major public health problem in developed and developing countries. Methods: This study was a cross sectional study in which 159 diagnosed hypertensive patient and 75 with normal blood pressure (normotensive) were enrolled for compare. These patients sought a through health cheek up including blood pressure assessment between May 2012 to April 2013 in Tairunnessa Memorial Medical College and Hospital. Lipid parameters total cholesterol (TC), triglyceride (TG), low density lipoprotein ( LDLc) and high density lipoprotein (HDLc) were estimated by enzymatic colorimetric test. Results: The mean of Systolic blood pressure and Diastolic blood pressure of hypertensive were higher than normotensive $(p<0.001)$. The serum levels of total cholesterol, triglyceride and $L D L-C$ in hypertensive subject were higher than normotensive and statistically significant $(p<0.001)$. Serum HDL-C was significantly lower $(p<0.001)$ in hypertensive patients than in normotensive. Age, waist circumference (WC), body mass index (BMI) showed significant association with hypertensive patients $(p<0.001)$ than in normotensive subjects.The logistic regression analysis indicates hypertensive were 1.2 times higher total cholesterol, 1.3 times higher triglyceride and 1.2 times higher LDL-C than normotensive and was statistically significant (p<0.001). HDL-C was 1.08 times lower in hypertensive than normotensive and statistically significant $p<0.001$ ). Conclusion: Analytical results of the study revealed that hypertensive patients have been found to have close association with dyslipidemia. Hypertension and dyslipidemia can be modified either by proper life style changes or medical management or by the combination of the both. This study suggests that hypertensive patients need measurement of blood pressure and lipid profile at regular interval to prevent heart diseases and stroke.
\end{abstract}

Key words: Hypertension, Lipid profile, Total cholesterol, Triglyceride, LDL-C, HDL-C.

Introduction:

Hypertension is a major public health problem in developed and developing countries. Hypertension and dyslipidemia are associated with oxidative stress and are major causes of cardiovascular disease amounting to $30 \%$ of global death rate. ${ }^{1}$ It is widely accepted that cardiovascular disease is associated with hypertension and increased blood levels of low-density lipoprotein (LDL), total cholesterol (TC), and triglycerides. In contrast, a low level of high density lipoprotein (HDL) is a risk factor for mortality from cardiovascular disease. ${ }^{2}$ Epidemiological studies have established a strong association between hypertension and coronary artery disease (CAD). Hypertension is a major independent risk factor for the development of CAD, stroke and renal failure. Non-communicable disease risk factor survey Bangladesh 2010 studies have estimated that among adults, the prevalence to range from $16 \%$ to $20 \%$. ${ }^{3}$ Nutrition, health and demographic survey of Bangladesh 2011 have estimated that among adults, the prevalence of hypertension was 34\%. Urban subjects had a higher prevalence of hypertension $37 \%$ while for rural prevalence was significantly lower at 33\% $(\mathrm{p}<0.001) .{ }^{4}$

The Seventh report of the Joint National Committee on Prevention, Detection, Evaluation and Treatment of High Blood Pressure (JNC-7) classifies BP as normal (Systolic $\mathrm{BP}<120 \mathrm{mmHg}$ and Diastolic $\mathrm{BP}<80 \mathrm{mmHg})$. Pre hypertension (Systolic BP 120-139 mmHg and Diastolic BP 80-89 mmHg), Hypertension stage I (Systolic BP 124159 mmHg and Diastolic BP 90-99mmHg and hypertension 
stage 2 (Systolic BP 160mmHg and Diastolic BP 100mmHg) respectively. ${ }^{5}$

Atherosclerotic diseases are a leading cause of disability and death worldwide and two third of these are associated with dyslipidemia. Worldwide, there is a wide variation in mean population lipid profiles levels. Increased serum total cholesterol (TC), triglyceride (TG), low density lipoprotein (LDL-c) level and decreased high density lipoprotein (HDLc) are associated major risk factors for cardiovascular disease (CVD). Dyslipidemia comprising altered ratio of high to total cholesterol level and isolated evaluation of the low density lipoprotein or triglycerides is usually associated with rising blood pressure levels. There are strong and relations between total LDL cholesterol concentrations and coronary heart disease risk. In Bangladesh consumption of saturated fat and red meat is injurious to health and a known risk factor for CVD especially hypertension. ${ }^{6}$

The purpose of the study was to compare the blood lipid levels in hypertensive patients with normotensive subjects and find out its association between hypertension and lipid profile.

\section{Methods:}

This study was a cross sectional study in which 159 diagnosed hypertensive patient and 75 with normal blood pressure (normotensive) were enrolled. These patients sought a through health cheek up including blood pressure assessment between May 2012 to April 2013 in Tairunnessa Memorial Medical College and Hospital. All the subjects were residents of the surrounding areas and aged 30-60 years. Patients with features of any cardiac or renal complication and major medical problem were excluded. After obtaining informed consent data collectors completed data sheet by interview, clinical examination, anthropometric measurement and investigation.

\section{Blood Pressure measurement:}

Blood pressure was measured after the subject had retested for at least 5 minutes from right arm placed at the heart level by a physician. Two measurements were taken by a mercury sphygmomanometer with at least 5 minutes between successive measurements. The mean of two measurements of Korotkoff phase I was recorded for systolic blood pressure (SBP). The mean of two values of korotkoff phase IV was recorded for diastolic pressure (DBP). Hypertension was defined as an average SBP $\geq 140$ $\mathrm{mmHg}$ and $\mathrm{DBP} \geq 90 \mathrm{mmHg}$ without antihypertensive medication. $^{1}$

\section{Serum assessments:}

Venous blood was collected in the morning after an overnight fast and serum was used for the biochemical tests. Lipid parameters (TC, TG, LDLc and HDLc) were estimated by enzymatic colorimetric test. Dyslipidemia was defined according to Evaluation and Treatment of High Blood Cholesterol in Adults. Executive Summary of the Third Report of the National Cholesterol Education Program (NCEP) Expert Panel on Detection, Evaluation and Treatment of High Blood Cholesterol in Adults (Adults Treatment Panel III). Hypercholesterolemia was defined as fasting total serum cholesterol and triglyceride of greater than or equal to $200 \mathrm{mg} / \mathrm{dl}$ and 150 respectively. Blood concentration of LDL-C (low-density lipoprotein cholesterol) equal or above $150 \mathrm{mg} / \mathrm{dl}$ and blood concentration of HDL-C (high-density lipoprotein cholesterol) under 40mg/dl respectively, were considered to be undesirable. ${ }^{7}$

\section{Results:}

In the present study, maximum numbers of patients of both sexes were between 41-60 years age and the percentage had declined sharply below theses ages. The mean age of hypertensive patients and normotensive were 47.67. \pm 1.11 vs 38.39. \pm 1.43 years' respectively and statistically significant (p-0.001). (The results are shown in Table 1 and 3).

Serum levels of total cholesterol, triglyceride and LDL-C in hypertensive subject were $238.31 \pm 2.17,178.34 \pm 1.94$ and151.28 $\pm 1.55 \mathrm{mg} / \mathrm{dl}$ respectively while in normotensive subjects the results were $187.01 \pm 4.26,141.48 \pm 2.48$ and $11031 \pm 2.33 \mathrm{mg} / \mathrm{dl}$ respectively. There were significantly $(p<0.001)$ higher levels in hypertensive patients. The serum HDL-C was significantly lower $(p<0.001)$ in hypertensive patients $41.24 \pm 1.07 \mathrm{mg} / \mathrm{dl}$ than in normotensive subjects $44.28 \pm 1.82 \mathrm{mg} / \mathrm{dl}$ respectively. (The results are shown in Table 2 and Fig 1).

The mean systolic blood pressure (SBP) of hypertensive and normotensive were 146.77....84 vs 119.21. $\pm 1.4 \mathrm{~mm}$ of $\mathrm{Hg}$ and mean diastolic blood pressure (DBP) was 98.92.土.72 vs $80.89 . \pm .86 \mathrm{~mm}$ of Hg. The mean of SBP and DBP of hypertensive was found to be higher than normotensive $(p<0.001)$. Age, waist circumference (WC), body mass index (BMI) showed significant association with hypertensive patients $(p<0.001)$ than in normotensive subjects. (The results are shown in Table 3 and Fig 2).

Binary logistic regression analysis showed total cholesterol was significantly associated with hypertensive patients Odds Ratio(OR) 1.20; 95\% CI (0.97-1.38), p<0.001. Triglyceride and LDL-C was significantly associated with hypertensive patients (OR 1.29; 95\% CI 0.91-1.41, p<0.001) and(OR 1.22; 95\% CI 0.89-1.31, p<0.001) respectively. HDL$\mathrm{C}$ was also associated with hypertensive patients (OR 1.08; 95\% CI (0.96-1.28), $\mathrm{p}<0.001$. (The results are shown in Table 4). 
Table-I

Age distribution of hypertensive and normotensive patients.

\begin{tabular}{lccccc}
\hline \multirow{2}{*}{$\begin{array}{l}\text { Age groups } \\
\text { (years) } \mathrm{n}(\%)\end{array}$} & \multicolumn{2}{c}{ Hypertensive $=159$} & & \multicolumn{2}{c}{ Normotensive $=75$} \\
\cline { 2 - 3 }$<=30$ & Male $(\mathrm{n}=88)$ & Female $(\mathrm{n}=71)$ & & Male $(\mathrm{n}=40)$ & Female $(\mathrm{n}=35)$ \\
$31-40$ & $12(80)$ & $3(20)$ & & $16(59)$ & $11(41)$ \\
$41-50$ & $18(51)$ & $17(49)$ & & $9(43)$ & $12(57)$ \\
$51-60$ & $24(53)$ & $21(47)$ & & $7(54)$ & $6(46)$ \\
$61-70$ & $21(49)$ & $22(51)$ & & $6(75)$ & $2(25)$ \\
$>=71$ & $9(75)$ & $3(25)$ & & $2(33)$ & $4(67)$ \\
\hline
\end{tabular}

$\mathrm{NF}^{*}=$ Not found any patients that had normal blood pressure. Age and HTN status are statistically significant at 5\%

Table-II

Serum lipid profile of hypertensive and normotensive patients.

\begin{tabular}{lcccccc}
\hline Indicators(mg/dl) & \multicolumn{2}{c}{ Hypertensive $=159$} & & \multicolumn{2}{c}{ Normotensive $=75$} & P-Value \\
\cline { 2 - 3 } & Mean (SE) & CI & & Mean (SE) & CI & \\
\hline Total Cholesterol & $238.31(2.17)$ & $221.01-242.5$ & & $187.01(4.26)$ & $181.52-191.51$ & 0.001 \\
Triglyceride & $178.34(1.94)$ & $171.51-181.1$ & & $141.48(2.83)$ & $138.82-143.13$ & 0.001 \\
HDL-cholesterol & $41.24(1.07)$ & $39.13-46.35$ & & $44.28(1.82)$ & $42.65-49.91$ & 0.001 \\
LDL - cholesterol & $151.28(1.55)$ & $148.23-155.3$ & & $110.31(2.33)$ & $107.65-114.96$ & 0.001 \\
\hline
\end{tabular}

Table-III

Characteristics of hypertensive patients and normotensive subjects

\begin{tabular}{|c|c|c|c|c|c|}
\hline \multirow[t]{2}{*}{ Indicators } & \multicolumn{2}{|c|}{ Hypertensive $=159$} & \multicolumn{2}{|c|}{ Normotensive $=75$} & \multirow[t]{2}{*}{ P - value } \\
\hline & Mean (SE) & CI & Mean (SE) & CI & \\
\hline Age (in years) & $47.67(1.11)$ & $45.48-49.86$ & $38.39(1.43)$ & $35.53-41.24$ & 0.001 \\
\hline Height (Meter) & $1.55(.01)$ & $1.53-1.57$ & $1.55(.01)$ & $1.53-1.57$ & 0.001 \\
\hline Weight (Kg) & $62.32(.97)$ & $60.41-64.23$ & $56.87(1.39)$ & $54.09-59.65$ & 0.001 \\
\hline *WC(cm) & $86.01(.89)$ & $84.25-87.76$ & 78.27(1.44) & $75.40-81.13$ & 0.001 \\
\hline $\mathrm{BMI}$ & $25.98(.44)$ & $25.10-26.85$ & $23.58(.51)$ & $22.58-24.59$ & 0.001 \\
\hline Systolic BP (mm Hg) & $146.77(.84)$ & $141.11-151.42$ & 119.21(1.4) & $117.42-23.01$ & 0.001 \\
\hline Diastolic BP (mm Hg) & $98.92(.72)$ & $94.49-101.36$ & $84.89(.86)$ & $81.17-87.61$ & 0.001 \\
\hline
\end{tabular}

${ }^{*}$ WC = Waist Circumference

Table-IV

Binary logistic results for hypertensive and normotensive patients.

\begin{tabular}{lccc}
\hline Indicators & Odds ratio & Confidence interval (CI) & P - Value \\
\hline Total Cholesterol $(<200)$ & 1.20 & $0.97-1.38$ & 0.000 \\
Triglyceride $(<150)$ & 1.29 & $0.91-1.41$ & 0.001 \\
Serum HDL-cholesterol $(60)$ & 1.08 & $0.96-1.28$ & 0.003 \\
LDL - cholesterol $(<100)$ & 1.22 & $0.89-1.31$ & 0.001 \\
\hline
\end{tabular}


Comparison and man values of SBP and DBP between hypertensive and normotensive patients

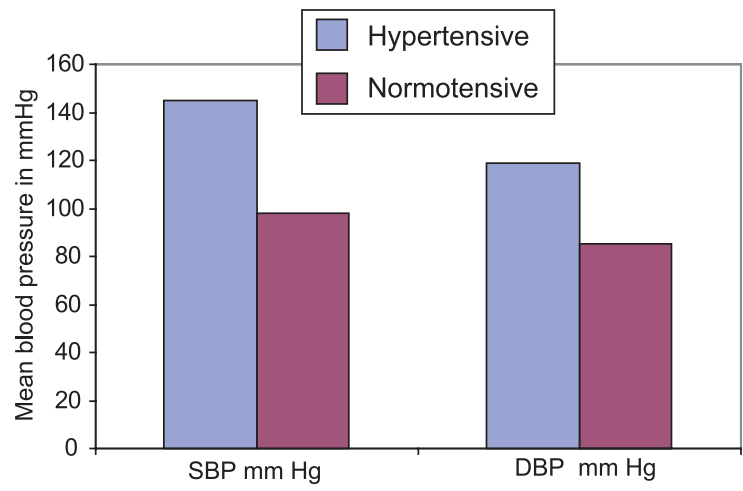

Fig.-1: Comparison and mean values for SBP and DBP between hypertensive and normotensive Subject.

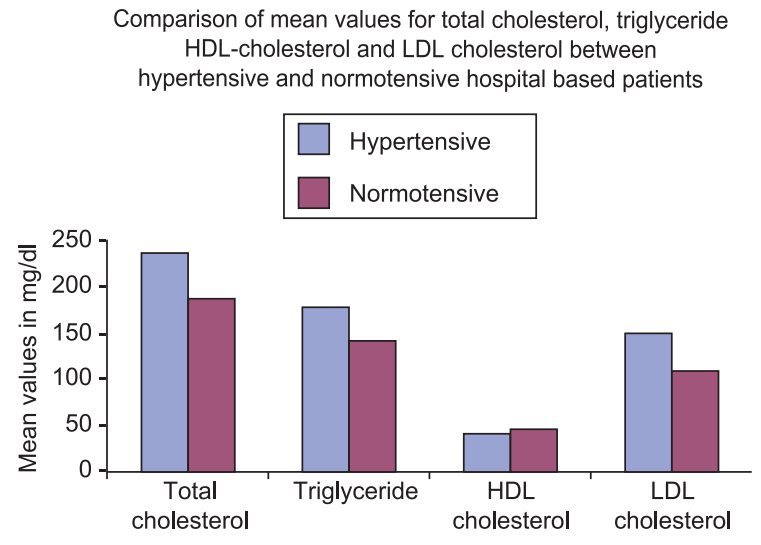

Fig.-2: Comparison of mean values for Total Cholesterol, Triglyceride, HDL-cholesterol and LDL - cholesterol between hypertensive and normotensive patients.

\section{Discussion:}

Hypertension is recognized globally as a major public health problem. ${ }^{8}$ It is known as the well known risk factor for coronary heart disease, type 2 diabetes mellitus and renal diseases. ${ }^{9}$ About $80 \%$ of hypertensive persons have co morbidities such as obesity, glucose intolerance, low HDL-Cho, high LDL-Cho and increased triglycerides etc. Two or more co morbidities are found in about more than 50\% hypertensive patients. Present study was focused on to study the lipid profile pattern of hypertensive patients with normotensive patients. In present study the results revealed that the mean value of serum total cholesterol, triglycerides and serum LDL-cholesterol was significantly higher and statistically significant. The mean of HDL of cases were lower than that of controls and was statistically significant. A prospective study which was based in the northern region of Bangladesh, to investigate the lipid profile status in hypertensive patients as compared to healthy normotensive controls. Their study revealed similar findings of high serum total cholesterol, triglycerides and LDL-cholesterol as observed in our study. 10 Our findings of increased level of total cholesterol in hypertensive patients are similar to the findings of some other studies. ${ }^{11}$ A prospective study conducted in Bangladesh on type 2 diabetes mellitus patients with and without hypertension revealed significantly high total cholesterol, triglycerides and LDL-cholesterol in hypertensive patients with type 2 diabetes mellitus as compared to the normotensive type 2 diabetes mellitus subjects. ${ }^{12}$ Few studies showed strong association of hypertension and dyslipidimia with major risk factors of coronary heart disease. ${ }^{13}$

Based on the results obtained from the present study, we concluded that serum lipid profile especially total cholesterol, triglycerides, LDL-cholesterol levels and HDLcholesterol are significantley associated with hypertension.

\section{Conclusion:}

Analytical results of the study revealed that hypertensive patients have been found to have close association with dyslipidemia. Hypertension and dyslipidemia can be modified either by proper life style changes or medical management or by the combination of the both. This study suggests that hypertensive patients need measurement of blood pressure and lipid profile at regular interval to prevent heart diseases and stroke.

\section{Acknowledgment:}

Authors are very grateful to all the participants for the study. The authors felt immense thanks to physicians, pathologists and pathological staff of Tairunnessa Memorial Medical College and Hospital.

\section{Reference:}

1. Treatment of hypertension in the prevention and management of Ischemic heart disease: A scientific statement from the American heart association council for high blood pressure research and councils on clinical cardiology and epidemiology and prevention, 2007;115:2761-2788. Htpp:/ /www.circulationaha.org. World Health Organization International society of hypertension Guidelines Subcomittee. J Hypertens 1999; 17: 151-183.

2. Criqui MH, Heiss G, Cohen R et al. Plasma triglyceride level and mortality from coronary heart disease. $\mathrm{N}$ Engl $\mathrm{J}$ Med 1993;328:1220-5. 
3. Non-communicable disease risk factor survey Bangaldesh 2010.

4. Nutrition, health and demographic survey of Bangladesh-2011.

5. MedicineNet. High Blood Pressure. http://www.medicinenet, com/high blood pressure/pages 5.htm.viewed on nov, 2004.

6. Applel LI, Moore TJ, Obarzanek ey al. for the DASH Collaborative research group Aclinical trail of the effects of diatary patterns on blood pressure. N Engl J med 1997; 336: 1117-24.

7. Expert Panel on Detection: Evaluation and Treatment of High Blood Cholesterol in Adults. Executive Summary of the Third Report of the National Cholesterol Education Program (NCEP) Expert Panel on Detection, Evaluation and Treatment of High Blood Cholesterol in Adults (Adults Treatment Panel III) JAMA 2011,185 (19):2486-97.

8. Cappuccio FP, Micah FB, Emmett L, Prevalence, detection, management and control of hypertension in Ashanti, West Afric. Hypertension. 2004; 43: 1017.

9. Gupta R Trends in hypertension epidemiology in India. J. Hum. Hypertens.2004; 18:73-8.
10. Ms Saha, Shaha NK et al. Serum lipid profile of hypertensive patients in northern region of Bangladesh. Journal of Biosciences.2006; 14: 93-97.

11. Shahadat H, Maliha R et al. Study of Serum lipid profile in essential hypertensive patients. Mym. Med J.1999;8(1):22-25.

12. Alam SM, Ali S. Deb K et al. Serum lipid profile in hypertensive and normotensive type 2 diabetes mellitus patients- a comparative study. Mym. Med J.2003; 1:13-16.

13. Joseph Osagie Idemudia, Emmanuel Ike et al. Plasma lipid profiles in hypertensives Nigerians. The internet Journal of Cardiovascular research. ISSN: 1540-2592. 6(2). DOI: 105580/117f.

14. Lakhsmankumar N, Deepthi J, Rao YN and Kiran Deedi M. Study of lipid profile, serum magnesium and blood glucose in hypertension. Biology and Medicine. 2010;2(1):6-16.

15. Lipid Research Clinic Programme: The lipid research clinics, coronary primary prevention trial results -2 JAMA 1984; 251:365-74.

16. Caroll MD, Lachev DA, Sorlie PD et al. Trends in serum lipids and lipoproteins of adults, J Amer Med Assoc 2005; 294:1773-81. 